\title{
TAKT PRODUCTION AS OPERATIONS STRATEGY: CLIENT'S PERSPECTIVE TO VALUE-CREATION AND FLOW
}

\author{
Joonas Lehtovaara ${ }^{1}$, Aleksi Heinonen ${ }^{2}$, Miika Ronkainen ${ }^{3}$, Olli Seppänen ${ }^{4}$, and \\ Antti Peltokorpi ${ }^{5}$
}

\begin{abstract}
Takt production is the most recent iteration of location-based production planning and control methods, adopting insights from lean construction and manufacturing operations management literature. In this research, we aim to advance the discussion between these domains further, especially considering the client's viewpoint. We approach takt production as a form of a project's operations strategy, allowing an explicit connection between client value-creation, production flow, and takt planning and control. Five key performance indicators are proposed to aid the client's understanding in assessing (and challenging) the effectiveness and value-creation capability of a specific takt production system. Furthermore, the approach is illustrated by applying it to a master planning phase of a large hospital project. The study has implications for clients and other stakeholders to evaluate their capability to operate with takt production from the lenses of valuecreation and production flow. We also hope that the study encourages scholars and practitioners to engage in further discussion with the nature of takt production, observing it from various theoretical and practical viewpoints.
\end{abstract}

\section{KEYWORDS}

Lean construction, takt production, operations strategy, production planning and control, production system design.

\section{INTRODUCTION}

Within the last decade, takt production has gained a rapid interest in construction operations management and in the lean construction community. Especially general contractors (GCs) have seen that the implementation of takt production leads to increased production performance. The documented benefits include radical duration reductions of over 50\% (e.g., Binninger et al. 2018), increased transparency of communication and effectiveness in production control (Frandson \& Tommelein 2014), increased quality and safety (Heinonen \& Seppänen 2016), and increased worker productivity (Kujansuu et al.

1 Doctoral Candidate, Department of Civil Engineering, Aalto University, Finland, joonas.lehtovaara@aalto.fi , orcid.org/0000-0002-4761-3811

2 Partner, Vison Oy, Finland, aleksi.heinonen@ vison.fi, orcid.org/0000-0003-2027-9646

3 Business Director, Vison Oy, Finland, miika.ronkainen@ vison.fi, orcid.org/0000-0002-3502-0488

4 Associate Professor, Department of Civil Engineering, Aalto University, Finland, olli.seppanen@aalto.fi, orcid.org/0000-0002-2008-5924

5 Assistant Professor, Department of Civil Engineering, Aalto University, Finland, antti.peltokorpi@ aalto.fi, orcid.org/0000-0002-7939-6612 
2020). Also, other project stakeholders, such as clients (e.g., Dlouhy et al. 2017a), have recently attempted to adopt the method into their practices. Here, client denotes the party responsible for funding and commissioning a construction project.

However, takt production research and development efforts have often been conducted through the lenses of GC and production (flow) effectiveness, with little focus on examining how takt production could most effectively increase value ${ }^{6}$ for the client. Even though the client evidently benefits from the increased production effectiveness (such as reduced duration), the missing client's value-driven key performance indicators (KPIs) hinder the possibility of transparently seeing and reaping all the potential benefits of takt production. For a GC, utilizing takt production successfully is an ambiguous promise as there are no specific and measurable requirements for what successful takt production is.

The client's viewpoint has been previously explored, for example, by Dlouhy et al. (2017a), Binninger et al. (2017a), and Haghsheno et al. (2016). In their studies, they rightly argue that the value-creation for the client should be complemented with an adequate process-driven approach. Simultaneously, the process design of takt production should be aligned with the client's value requirements, for example, by recognizing the need to determine takt time and production phasing based on demand and clientdetermined milestones. Here, takt time serves as a nominator between demand and supply, pacing the production to match customer needs.

Now, we would like to expand these ideas on continuing to build a bridge between the client's value-creation and production flow effectiveness. In this study, we do this by approaching takt production as a form of a project's operations strategy, adopting viewpoints from manufacturing operations management literature, such as from Factory Physics (Hopp \& Spearman 2011). We aim to clarify how the client's success can be connected to takt production system's performance through tangible KPIs: this would allow clients to evaluate how different takt production decisions could most successfully promote their project and long-term business goals, to proactively advance flowefficiency improvement, and also to better understand how capable different service providers, such as contractors, are to succeed with takt production.

The remaining of the paper is structured as follows. First, we look at how takt production could be inspected from the operations strategy perspective. We then propose KPIs that connect value-creation and flow-effectiveness to takt planning and control process. Then, we demonstrate the approach in action through a case example of a takt planning process in a master planning phase of a large Finnish hospital project. Finally, we engage in a brief discussion regarding the implications of the approach and conclude with possible future research avenues.

\section{OPERATIONS STRATEGY APPROACH}

In the last three decades, the transformation-flow-value (TFV) (Koskela 1992) theory of construction production has guided the research of planning and control methods towards pursuing flow-efficiency among lean construction research. The research and development of takt production have followed the same path. Indeed, documented cases have reported takt production to improve production flow holistically, especially by

\footnotetext{
${ }^{6}$ We here follow the definition of Womack \& Jones (1996); they define value as something determined by the client, being "a specific product (a good or service, and often both at once) which meets the customer's need at a specific price at a specific time".
} 
promoting good process flow elements such as minimized durations and minimized work in progress (WIP) (e.g., Linnik et al. 2013). As construction processes possess a large amount of waste that is often hidden under overly large time buffers (Ballard \& Howell 1998), aggressively focusing on flow-efficiency often has primarily positive effects, leading, for example, to a possibility to reduce waste and to reduce production duration.

While the sole pursuit of flow can be seen as somewhat valuable in itself, only promoting flow can be an inefficient goal if the initiative is not tied to value-creation and the project's desired scope (Pound et al. 2014, Koskela 1992). For example, reducing WIP to an absolute minimum might not result in increased benefits: instead, this reduction would most likely result in a high need for control efforts and WIP starvation, leading to increased costs and reduced performance (Little \& Graves 2008). Thus, with no connection to value-creation, solely improving flow might have certain initial benefits but can eventually face diminishing returns (Modig \& Åhlström 2012).

In takt production domain, lack of universal capability KPIs hinders contractors' ability to improve their flow-efficiency in a way that is connected to clients' goals. Construction projects form loosely coupled and fragmented systems, in which the production planning and control process are often procured as a "black box" from the contractors (e.g., Dubois \& Gadde 2002), often resulting in vague requirements for production performance that are based on guesses and rule of thumbs. The cycle continues as even though production performance can be improved within a project, this information seldom reaches the upstream and the client in the form that could help them improve their requirements for the next project (Henderson et al. 2013).

To shed light on this problem, we take a stance to approach takt production as a project's operations strategy, which considers how to conduct operations in a way that supports the prevailing business case best as possible. We adopt the definition of Pound et al. (2014), as they describe operations strategy as an act of designing, implementing, and controlling the portfolio of demand, time, cost, inventory, variability [with adequate buffer management], and capacity to best achieve a company's financial and marketing goals. By applying this definition to takt production, one can design, implement, and control a takt production system that resonates with the project scope and considers the improvement of flow-effectiveness. In the following section, we propose five KPIs to evaluate the effectiveness and value-creation capability of a takt production system and break down how individual components of the system are connected to the KPIs.

\section{TAKT PRODUCTION AND OPERATIONS STRATEGY}

\section{Project SCOPE (DEMAND)}

We propose the following KPIs for projecting the project scope and for roughly framing the desired product: total gross area [m2] and required quantity of work per gross area $[\mathrm{h} / \mathrm{m} 2]$. Quantity of work per gross area is the amount of work per gross area (e.g., sqm2 of drywall per gross area, acquired from quantity estimations), multiplied by productivity factor (e.g., how long does it take to produce a sqm2 of drywall, acquired from managers' and workers' experienced guesses, previous projects' data, or from public productivity ratio databases). The hours denote total working hours, and the crew sizes are determined separately. Even though the quantity varies between tasks and different project types, it provides a rough but easily comparable indicator for projects with similar scope. 


\section{TIME AND COST}

The third KPI, lead time, indicates how fast the whole production can be completed from start to finish and matched with client demand (Hopp \& Spearman 2011). Short lead time means that the client acquires the product faster while getting more swift returns for their investment. The fourth proposed KPI, batch-specific lead time, indicates how fast a specific part of production (i.e., an apartment) is completed from start to finish. Shortened batch-specific lead time can be beneficial when the client benefits from paced handover (Dlouhy et al. 2017b), enabling the commission of spaces before the whole product is finished. To decrease (batch-specific) lead times, minimizing variability, non-valueadding activities, and unnecessary (time)buffers is crucial. Together, these two indicators express how timely the production system can respond to the client's needs. Moreover, when the marginal cost for one day of production is known, one can deduce the value of lead time for the client and connect that to the costs. The marginal cost calculation should consider time-related costs of production and opportunity costs of the building operations.

\section{QUALITY AND TRANSPARENCY (INVENTORY)}

Transparency of the process and quality of the product increase when information flows through the production organization swiftly, urging the participants to steer the process and solve emerging issues proactively. By increasing production transparency, possibilities for errors, need for rework, and decreased value are reduced, driving for better quality. These benefits can be connected to a setting where the inventory of work is managed effectively and tightly: the work is progressed within small batches, with small WIP, crews working closely together, problems made actively visible and proactively solved (Hopp \& Spearman 2011).

Based on the notions above, we propose production's tightness, measured as the average area occupied by a single worker [m2/worker], as a fifth KPI. Tightness is closely connected to effective management of inventory, and when the cost of (poor) quality (such as costs of errors and rework) is known, one can derivate the value of tightness for the client. However, too tight work areas might yield diminishing results when the worker productivity decreases due to congestion (Thomas et al. 2006). Similar to the quantity of work per gross area, the production's tightness also provides a rough but comparable metric between similar types of projects.

\section{VARIABILITY}

Production variability indicates how much specific production metrics can deviate from their target amount. Accompanied with waste elimination, managing variability is central in enabling good production flow. In an ideal state all waste and variability are removed, however, in reality all production processes possess at least some degree of waste and variability (Hopp \& Spearman 2011), resulting in decreased stability and predictability of the production. To diminish the remaining variability's adverse effects, a portfolio of time, plan, inventory, and capacity buffers can be introduced to the process (Hopp \& Spearman 2011, Frandson et al. 2015). As variability can have several different sources and forms, flexible usage of the whole buffer portfolio is essential. The buffers can be implemented first during the process design and then applied as needed during production. Buffers also have varying effects on the presented KPIs, examined below. 


\section{Time buffering}

Time buffering prevents clashes of work crews and is useful when the process has a great amount of uncontrollable variability but results in longer lead times while hiding problems and hampering transparency (Horman \& Thomas 2005). In construction, time buffers are often used as a primary production balancing buffer.

\section{Plan buffering}

Denotes moving tasks from the main schedule to a 'secondary schedule' executed with spare time and resources. Reduces trade crews' idle time if they otherwise would have to wait for work, but an excess amount of backlog can result in increased lead time and ineffective inventory management, as the work is moved from the main schedule to more non-critical tasks and the problem-solving can be more easily avoided.

\section{Batch sizing (inventory buffering)}

Inventory buffering results in conducting work in larger batch sizes. This leads to an increased amount of simultaneously operated space and tasks, leading to increased WIP. Like time buffers, large inventory allows to prevent clashes between trades and brings flexibility to their work, but simultaneously results in longer lead times with decreased transparency (Little \& Graves 2008). With small inventory buffers, the production is observed in tighter cycles, resulting in transparency and urgency to improve the process more proactively. However, a small inventory can also create vulnerability when the system possesses a large amount of uncontrollable variability.

\section{Capacity buffering}

Denotes adding more resources to a task than what is necessarily needed. Enables to tackle problems within production proactively, as the excess capacity can be used for solving problems, quality management, and continuous improvement, however, can result in increased costs (Horman \& Thomas 2005). Capacity buffers theoretically decrease resource efficiency but increase it over time as problems are proactively solved. In construction, capacity buffers are often avoided as they are believed to increase initial costs.

\section{DESIGN, IMPLEMENTATION, AND CONTROL OF THE PRODUCTION SYSTEM}

\section{Takt planning (process design)}

Takt planning process consists of balancing buffer portfolio with takt production parameters, namely takt trains and wagons (process and sub-processes consisting of sequenced work tasks), takt time (time given to complete any set of sub-processes in a given location), and takt areas (determined locations in which work tasks are completed within the rhythm of takt time) (Lehtovaara et al. 2021). The selection of these parameters should be based on providing the best combination of client value and production effectiveness; takt planning is not just a method to create a visual plan but also to translate customer need into operative targets. In addition, the process design (and control) should consider the management of capacity (e.g., workers, material, equipment).

\section{Takt control (implementation and control)}

Takt control concerns how to implement, measure, and maintain the production system's desired performance. When the planned parameters are also maintained during the production, the desired value is achieved. To steer the production, takt control employs rhythmic production management aiming for steady wagon handoffs, proactive quality 
management, and visual, collaborative problem solving with root-cause analysis (Frandson et al. 2015). Production can also be steered by adjusting the takt production parameters (Binninger et al. 2017b) and applying or removing buffers. Together, these actions form a basis to steer the production so that the project's scope is meaningfully fulfilled while also providing a transparent way to communicate the production progress for all project stakeholders.

Figure 1 synthesizes the above-described elements, providing insights on the connection between the desired client value, process design, implementation and control, variability management, and the KPIs. It should be noted that the links are somewhat exaggerated as the system always needs to be inspected holistically. Next, we will demonstrate the presented approach in action through a case example.

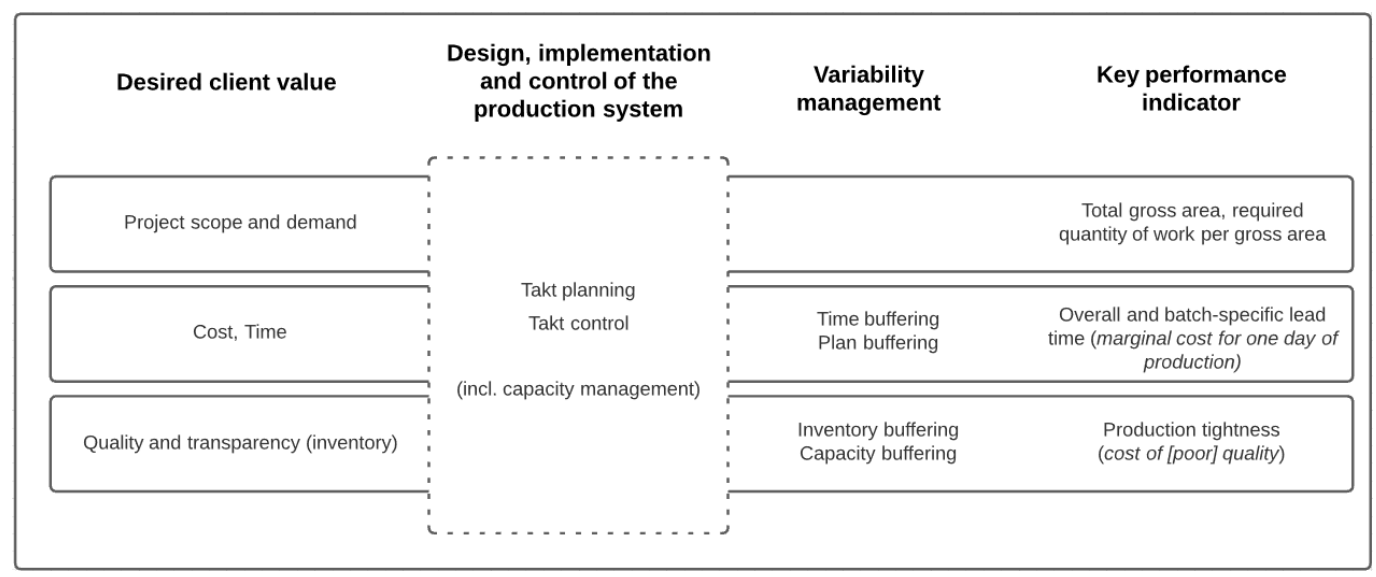

Figure 1: Synthesis of the operations strategy approach

\section{ILLUSTRATIVE CASE EXAMPLE}

\section{CASE DESCRIPTION}

The presented case is a study for a large hospital project in Helsinki, Finland. The project includes construction of new buildings and renovation of old spaces, consisting of $150,000 \mathrm{~m} 2$ of space. The project's overall duration is preliminarily estimated to span eight years and to cost $700 \mathrm{M} €$. The project is executed with an integrated project delivery method (IPD), in which the integrated project team concurrently performs design and production planning activities. The client has requested potential GC's to employ a takt production approach to seek lead time reduction opportunities and increase transparency during production.

The operations strategy approach was utilized in the master planning phase. The aim was to create a master plan that forms a rough but tangible frame to further help the IPD team conduct target-value-based production preparation. More specifically, the master planning phase's scope was to ensure a basis for transparent, stable, flow-efficient project execution that would provide the best client value. The master planning consisted of three steps, described below.

\section{SteP 1: Data Collection}

The following data were obtained from the preliminary designs, construction manager consultant estimations, references from previous similar projects, and from a national productivity factor database: 
was to obtain rough boundaries for the production rhythm and initial capacity requirements, in which the desired client value is connected to the production flow. Based on these boundaries, the plan will be refined and detailed concurrently with the contractors and other stakeholders in the later planning phases.

\section{Step 3: Iteration and Visualization of the Plan}

After formulating the initial plan, the planning parameters (takt time, amount and size of takt areas, number of wagons) were iterated reciprocally with adjusting the tightness, capacity, and (batch-specific) lead times. Inventory buffers were included by employing relatively sparse tightness ratios (75-120 $\mathrm{m} 2$ / worker), and capacity buffers were included by employing a conservative productivity factor. In the master plan, time and plan buffers were mostly avoided, however, some time buffer was scheduled between exterior and interior phases to ensure smooth transitions.

Through iteration, takt planning resulted in a master plan connected to the desired client value, illustrated in Figure 4. In addition to taking into account the value-creation, the parameters were balanced so that all the phases and functional areas could proceed in the same rhythm with little resource fluctuation, enabling smooth production flow. The plan is visualized with a standard takt plan visualization, even though the large number of zones and wagons may make it seem similar to flowline visualization. Nevertheless, the approach differs from other location-based methods (such as the Location-Based Management System) in a way that takt production considers size and number of (takt) areas, as well as the crew composition in takt wagons as flexible parameters, with capacity buffers used as the preferred buffer (Frandson et al. 2015).

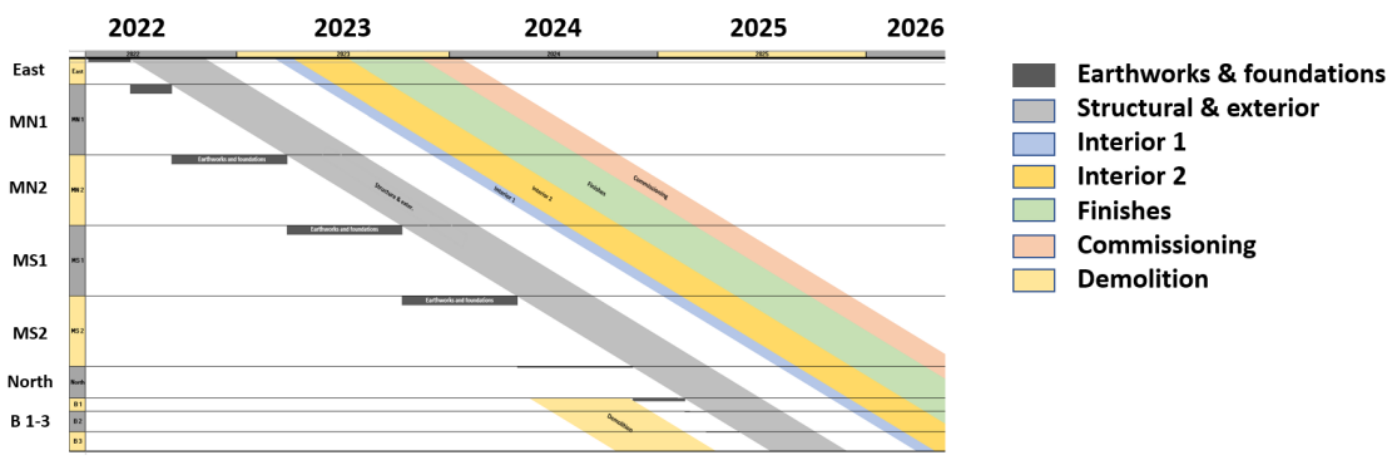

Figure 4: The master takt plan visualization

In summary, the master planning phase resulted in a takt plan that employs a balanced flow between the different buildings and work phases that vary in size and scope. The desired duration, which was a few years less than estimated beforehand, was also achieved in the plan. The resulting plan provides a solid basis for detailed, concurrent target-value-based design and production planning, in which the IPD team further investigates and iterates the presented plans. However, as the case is still in progress, the examination of detailed takt planning and control is left for future research. Hypothetically, the process could be carried on with a similar approach to the norm and micro levels of TPTC (takt planning takt control; Dlouhy et al. 2016) method.

\section{DISCUSSION \& CONCLUDING NOTES}

In this study, we approached takt production from the lenses of the project's operations strategy, allowing an explicit connection between client value-creation, production flow, 
and takt planning and control. We hope that the presented approach encourages scholars and practitioners to engage in further discussion with the nature of takt production, observing it from various theoretical and practical viewpoints.

The proactive role of a client helps to put customer value in the center of takt production. In an optimal situation, the client would drive the takt production process and development by assessing the proposed KPIs to form performance requirements, which would guide (general) contractors in designing and controlling the production system. Over the projects, the client should have an increased understanding of what level of value-creation the contractors can provide in certain settings, helping the clients assess demand rates for the projects that are realistic but tight enough to drive contractors to improve their operations management capabilities. Methods to improve these capabilities can be found in other studies, such as takt maturity development by Lehtovaara et al. (2020).

As illustrated through the case example, the approach helped the production planners to justify their decisions in the light of value-creation while providing a solid basis for further collaborative iteration and transparent communication of the plan. However, the case only illustrated the approach in the master planning phase, and future research should address its performance during detailed planning and production phases. We hypothesize that the approach could lead to increased performance within individual takt projects and more efficient long-term improvement; however, this needs validation in future studies. Future research should also address the validation of the proposed KPIs; the focus of the approach and the KPIs was on the value created by the operative functions, however, the value of other stakeholders, such as designers, could also be considered in future development. Moreover, data gathering for these metrics for different takt production use cases is needed to help clients and contractors effectively estimate and compare their projects' success.

\section{ACKNOWLEDGMENTS}

We would like to gratefully thank HUS Kiinteistöt for their help in advancing the implementation of the presented operations strategy approach.

\section{REFERENCES}

Ballard, G. and Howell, G. (1998). "What kind of production is construction." Proceedings (IGLC 6). Guarujá, Brazil.

Binninger, M., Dlouhy, J., \& Haghsheno, S. (2017a). "Technical takt planning and takt control in construction." Proceedings (IGLC 25). Heraklion, Greece.

Binninger, M., Dlouhy, J., Steuer, D., \& Haghsheno, S. (2017b). "Adjustment Mechanisms for Demand-oriented Optimisation in Takt Planning and Takt Control." Proceedings (IGLC 25). Heraklion, Greece.

Binninger, M., Dlouhy, J., Müller, M., Schattmann, M., \& Haghsheno, S. (2018). "Short Takt time in construction-a practical study." Proceedings (IGLC 26). Chennai, India. Dlouhy, J., Binninger, M., Oprach, S., \& Haghsheno, S. (2016. "Three-level method of Takt planning and Takt control-A new approach for designing production systems in construction." Proceedings (IGLC 24). Boston, USA.

Dlouhy, J., Binninger, M., Weichner, L. \& Haghsheno, S. (2017a). "Implementation of Lean Construction in Client Organizations - an Analysis of the Status Quo in Germany." Proceedings (IGLC 25). Heraklion, Greece. 
Dlouhy, J., Grobler, W., Binninger, M. \& Haghsheno, S. (2017b) "Lean Equipment Installation - Potentials of Using Takt Planning." Proceedings (IGLC 25). Heraklion, Greece.

Dubois, A., \& Gadde, L. E. (2002). "The construction industry as a loosely coupled system: implications for productivity and innovation." Construction Management \& Economics, 20(7), 621-631.

Frandson, A., Seppänen, O., \& Tommelein, I. (2015). "Comparison between location based management and takt time planning." Proceedings (IGLC 23). Perth, Australia.

Frandson, A., \& Tommelein, I. (2014). "Development of a takt-time plan: A case study." Construction Research Congress 2014. May 19-21, Atlanta, USA.

Haghsheno, S., Binninger, M., Dlouhy, J., \& Sterlike, S. (2016). "History and theoretical foundations of takt planning and takt control." Proceedings (IGLC 24). Boston, USA.

Heinonen, A., \& Seppänen, O. (2016). "Takt time planning in cruise ship cabin refurbishment: Lessons for lean construction." Proceedings (IGLC 24). Boston, USA.

Henderson, J. R., Ruikar, K. D., \& Dainty, A. R. (2013). "The need to improve doubleloop learning and design-construction feedback loops." Engineering, Construction and Architectural Management, 20(3), 290-306.

Hopp, W. J., and Spearman, M. L. (2011). Factory physics. Waveland Press.

Horman, M. and Thomas, H. (2005) "Role of inventory buffers in construction labor performance." Journal of Construction Eng. and Mgt. 131(7), 834-843

Koskela, L. (1992). "Application of the new production philosophy to construction." CIFE Technical Report Vol. 72. Stanford university.

Kujansuu, P., Lehtovaara, J., Salerto, S., Seppänen, O., \& Peltokorpi, A. (2020). "How Does Takt Production Contribute to Trade Flow in Construction?" Proceedings (IGLC 28). Berkeley, USA.

Lehtovaara, J., Heinonen, A., Lavikka, R., Ronkainen, M., Kujansuu, P., Ruohomäki, A., Örmä, M., Seppänen, O. \& Peltokorpi, A. (2020). "Takt Maturity Model: From Individual Successes Towards Systemic Change in Finland." Proceedings (IGLC 28). Berkeley, USA.

Lehtovaara, J., Seppänen, O., Peltokorpi, A., Kujansuu, P., \& Grönvall, M. (2021). 'How takt production contributes to construction production flow: a theoretical model." Construction Management and Economics, 39(1), 73-95.

Linnik, M., Berghede, K., \& Ballard, G. (2013). "An experiment in takt time planning applied to non-repetitive work." Proceedings (IGLC 21). Fortaleza, Brazil.

Little, JC and Graves, S. (2008) "Little's Law." In: Chhajed, D. and Lowe, T. (eds.) Building Intuition, International Series in Operations Research \& Management Science, New York: Springer, 81-100.

Modig, N., \& Åhlström, P. (2012). This is lean: Resolving the efficiency paradox. Rheologica.

Pound, E. S., Bell, J. H., \& Spearman, M. L. (2014). Factory Physics for Managers: How Leaders Improve Performance in a Post-Lean Six Sigma World. McGraw-Hill.

Thomas, H. R., Riley, D. R., \& Sinha, S. K. (2006). "Fundamental principles for avoiding congested work areas-A case study." Practice Periodical on Structural Design and Construction, 11(4), 197-205.

Womack, J. P., \& Jones, D. T. (1996). "Beyond Toyota: how to root out waste and pursue perfection." Harvard business review, 74(5), 140-172. 